\title{
Interfaces
}

INTERFACES Image Texte Language

$46 \mid 2021$

Jeux de Formats (2)

\section{Du format normatif et rectangulaire de la toile à sa déconstruction}

\section{Frédéric Montégu}

\section{(2) OpenEdition}

1 Journals

Édition électronique

URL : https://journals.openedition.org/interfaces/3572

DOI : 10.4000/interfaces.3572

ISSN : 2647-6754

Éditeur :

Université de Bourgogne, Université de Paris, College of the Holy Cross

Référence électronique

Frédéric Montégu, "Du format normatif et rectangulaire de la toile à sa déconstruction », Interfaces [En ligne], 46 | 2021, mis en ligne le 15 décembre 2021, consulté le 18 juin 2022. URL : http:// journals.openedition.org/interfaces/3572 ; DOI : https://doi.org/10.4000/interfaces.3572

Ce document a été généré automatiquement le 18 juin 2022.

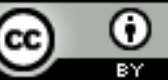

Les contenus de la revue Interfaces sont mis à disposition selon les termes de la Licence Creative Commons Attribution 4.0 International. 


\title{
Du format normatif et rectangulaire de la toile à sa déconstruction
}

\author{
Frédéric Montégu
}

\section{Introduction}

1 Rarement questionné, le dispositif iconique et rectangulaire informe la plupart des images qui font notre actualité (photographies, cinéma, écrans, publicités...). La présupposition de cette forme quadrangulaire semble avoir son origine dans l'instauration, à la Renaissance italienne, du tableau moderne ou quadro ${ }^{1}$. Verticalité, planéité, frontalité : le cadre rectangulaire conditionne notre réception de l'image et notre vision du monde. Cette géométrie définissant la surface de l'image est traditionnellement redoublée par le cadre/objet ${ }^{2}$ qui délimite une sorte de templum exclusif, faisant de la représentation un objet de contemplation. Dès lors, nous proposons ici de mieux comprendre pourquoi et comment cette matrice du format rectangulaire devient un des éléments spécifiques de l'image. Pourquoi y-a-t-il une déconstruction effective de cet objet/toile 3 à partir du XX siècle ? Comment les artistes arrivent-ils aussi, à partir des années 1960, à détourner la géométrie de la toile, à faire en sorte que la matérialité géométrique du format soit rendue visible? De fait, quels sont les enjeux, à la fois formels et sémantiques, de cette structure spécifique qui existe maintenant depuis près de six siècles?

\section{Cadre rectangulaire de la toile (peinture)}

Le cadre/objet est un des éléments plastiques de l'image, et il a pour particularité d'en être un élément caractéristique, comme la composition spatiale qui lui est liée. Il est spécifique car c'est lui qui isole l'image, qui la circonscrit, qui la désigne en tant qu'image. Le cadre a en effet longtemps été considéré, selon la définition de Meyer Shapiro, comme une "clôture régulière isolant le champ de la représentation, de la surface environnante»(13). Dès 1435, Leon Battista Alberti désigne «la peinture 
comme une fenêtre dont il est nécessaire de tracer d'abord le cadre. La bordure fonctionne dès l'origine, dans cette première théorisation du genre pictural, comme un signal qui indique au spectateur qu'il se trouve non pas face au réel, mais devant une œuvre née de la mimésis » (Goetz). Martine Joly écrit à propos du cadre :

[...] (de la photographie à la page de magazine, en passant par la peinture, l'écran de cinéma, de télévision ou d'ordinateur etc ...); il est une limite, une frontière physique qui délimite et sépare deux espaces distincts qui sont l'espace représenté (à l'intérieur du cadre) et l'espace de l'exposition ou de monstration (à l'extérieur du cadre, le hors-cadre). (109)

3 Le cadre rectangulaire qui entoure la majorité de nos images ${ }^{4}$ est rarement un objet de questionnement car il semble aller de soi. Pourtant il ne correspond pas à la vision humaine sphérique et floue au bord. Ce cadre est donc avant tout une construction culturelle, mais aussi matérielle. "Pourquoi nos représentations sont-elles majoritairement délimitées par un cadre rectangulaire? Une continuité semble manifeste entre le cadre rectangulaire qui prévaut aujourd'hui majoritairement et l'héritage perspectif de nos images » (Charbonnier 19).

4 Le cadre, dont nous avons dit qu'il était le plus souvent rectangulaire, est un héritage particulier de la Renaissance italienne et de la représentation en perspective (le fait de donner l'illusion d'un creusement perspectif). Le rectangle sert de repère et de guide pour construire les lignes de fuite et l'illusion de la troisième dimension, c'est-à-dire la profondeur. Selon Isabelle Cahn :

À l'origine de ce renouvellement, on trouve d'abord Brunelleschi et Alberti, puis Dürer, qui mirent successivement au point des appareils facilitant le tracé de l'espace perspectif. Le dispositif en fut sans cesse retouché par les artistes : 4 siècles plus tard, Van Gogh utilisait encore un modèle de sa fabrication qui lui permettrait de dessiner, grâce à des repères géométriques servant à établir les lignes de fuite et les propositions. (56)

5 Louise Charbonnier, de son côté, distingue à juste raison la coupure sémiotique du cadre/objet. En effet la forme rectangulaire de la toile sur châssis produit un champ de vision spécifique. Cette dite "coupure sémiotique» est une découpe formelle (signifiant) entraînant des enjeux sémantiques (signifiés) :

La coupure sémiotique comme geste premier du dispositif iconique délimite d'abord un champ de vision en instituant un ordre dans le chaos, rappelant ainsi le champ de vision ou templum que traçait l'augure dans le ciel pour y pratiquer la divination. Ce champ de vision est aussi champ de signification où l'on peut lire et interpréter les signes qui s'y inscrivent (passage d'oiseaux ou peinture). (29)

6 Puis intervient le cadre lui-même, ou plutôt le cadre/objet qui semble notamment redoubler la coupure sémiotique opérée par le cadre rectangulaire. Cet objet spécifique autour de la peinture a pour fonction de protéger mais aussi de clôturer, de borner cet espace de la diégèse ; il a donc une fonction de monstration et en même temps de séparation puisqu'il dissocie - et ce d'une manière effective - l'espace diégétique (la peinture) de l'espace réel (le musée, ou plus largement, le lieu d'exposition). Étienne Souriau définit le « cadre » comme suit :

Bordure, entourage, étymologiquement de forme carrée (cadre vient de l'italien quadro, c'est-à-dire objet carré, du latin populaire quadrum), mais le plus souvent rectangulaire [...]. Le cadre est une sorte de bordure dans laquelle on place un tableau, une photographie, un bas-relief ... bref, une œuvre à deux dimensions [...]. Mais il a aussi et surtout d'importantes fonctions esthétiques. Il circonscrit l'œuvre et appuie son caractère de monde autonome (comme le disait Baudelaire, un cadre « ajoute à la peinture... - je ne sais quoi d'étrange et d'enchanté - En l'isolant de 
l'immense "nature »). Il délimite l'œuvre par un contour net qui en précise la

forme d'ensemble. Il ménage une transition de l'œuvre et son entourage. (295-296)

\section{Tableau}

7 Paradoxalement, le cadre précise la forme d'ensemble, c'est-à-dire la forme rectangulaire du tableau et, par sa transition effective, fait oublier cette forme spécifiquement géométrique. Tout l'enjeu de la Renaissance est d'ailleurs basé sur cela : faire oublier la configuration bidimensionnelle et rectangulaire du tableau. Les florentins $\mathrm{du} \mathrm{XV}^{\mathrm{e}}$ siècle mettent au centre de leur dispositif théorique «le principe d'une fidélité spéculaire au monde visible. Précision, reproduction: un tableau est réussi quand il imite à "s'y méprendre » les apparences extérieures». (Clay 9) On le compare à une fenêtre ouverte sur le monde. Pour Leon Battista Alberti ${ }^{5}$,

La fonction du peintre consiste à circonscrire et à peindre sur un panneau ou un mur donné, au moyen de lignes et de couleurs, la surface visible de toute espèce de corps, de sorte que, vu à une certaine distance et sous un certain angle, tout ce qui sera représenté apparaisse en relief et ait exactement l'apparence de ce corps même. (209)

8 L'illusionnisme de la Renaissance fait donc oublier le cadre rectangulaire de la toile, sa forme géométrique et bidimensionnelle. Nous pourrions néanmoins repérer des peintures non rectangulaires, par exemple les tondos à la Renaissance, mais ces derniers restent tout de même très minoritaires. Pendant près de cinq siècles, nous assistons à la fois à une omniprésence de cette structure issue du cadre, et en même temps à son oubli, puisqu'elle n'est considérée, en fait, que comme le cadre fictif d'une fenêtre : à la fois omniprésence et « oubli » de la toile/rectangle. Les spectateurs ne regardent plus la forme géométrique puisqu'elle est devenue une répétition récurrente, une norme spécifique de l'image diégétique; ils regardent directement la narration picturale, le degré de ressemblance par rapport à la nature, l'illusion de la troisième dimension. Plus l'illusionnisme est efficace, plus le spectateur s'identifie à la diégèse du champ de l'image, et plus la couture sémiotique est oubliée.

9 Prenons un exemple spécifique de la Renaissance italienne, Saint Jérôme dans un intérieur d'Antonello de Messine (1456) ${ }^{6}$. Dans cette œuvre, nous voyons apparaître une fine construction en perspective, notamment l'encadrement de la scène par une architecture en forme de portail qui redouble l'effet de cadrage afin de mettre en évidence la scène elle-même. Les formes gothiques de cette architecture (ou plutôt sa représentation) ainsi que les détails proches de la nature morte, comme la coupe sur la première marche, le carrelage, ou la vue du paysage au fond de la pièce, renvoient sûrement au fait qu'Antonello de Messine avait étudié les peintres hollandais, peignant à la même époque.

10 Cette scène est un véritable trompe-l'œil, un parfait illusionnisme au regard de la réalité. Très vite, le spectateur s'identifie à la diégèse proposée du fait, à la fois, de la représentation en perspective très aboutie, et de la narration évoquée: dans un intérieur se dresse un imposant meuble de bois, qui est en fait le cabinet d'étude de Saint-Jérôme. Le peintre, par l'intermédiaire de cette peinture, nous expose donc l'histoire du personnage en question : Saint-Jérôme. Docteur et Père de l'Eglise des IV et $\mathrm{V}^{\mathrm{e}}$ siècles après $\mathrm{J}-\mathrm{C}$, Saint-Jérôme est reconnaissable ici grâce à la tenue pourpre de cardinal. De plus, Jacques de Voragine, dans La Légende dorée, lui attribue l'extraction d'une épine de la patte d'un lion; or, nous voyons apparaître dans cette peinture 
l'ombre d'un lion, à droite du meuble. Mais surtout, Saint-Jérôme est un moine lettré, auteur de la vulgate, la traduction latine officielle de la bible; il est présenté ici de profil, comme un savant absorbé dans sa lecture, au milieu des ouvrages qui garnissent ses étagères ; tous ces éléments suggèrent son activité d'exégète biblique.

La diégèse du champ de cette image est très efficace, et nous fait oublier la couture sémiotique, d'autant que les fenêtres du fond et la vue du paysage garantissent l'efficacité du creusement perspectif.

\section{Visibilité du format rectangulaire de la toile}

12 La période classique va imposer un peu plus la forme rectangulaire de la toile, en tant que norme spécifique, et les châssis non rectangulaires se feront de plus en plus rares. De plus, le cadre/objet qui entoure la toile va devenir un objet mobilier à part entière et prendre une certaine ampleur, à la fois dans sa lisibilité, et dans sa reconnaissance sociale et culturelle. Il existe, par exemple, pour le XVIII ${ }^{\mathrm{e}}$ siècle, des cadres portant les illustres marques de Caffieri, Legoupil, ou EEben. Mais la toile sur châssis, comme le cadre/objet vont être totalement bouleversés, à la fois d'un point de vue esthétique et social, par ladite Modernité à la fin du XIX ${ }^{\mathrm{e}}$ siècle. En effet, la matérialité géométrique de la toile va être rendue de plus en plus lisible, alors même que le cadre/objet va tendre, au contraire, à disparaître au fur et à mesure.

13 À partir du moment où il y aura une mise en évidence de la couture sémiotique dans la peinture, à la fin du XIXe début du XXe siècle, l'identification à la narration, à l'aspect diégétique de l'image, ne se fera plus d'une manière aussi évidente. La géométrie rectangulaire étant mise en évidence, la déconstruction du format se fera d'abord par une autoréflexivité de la surface carrée ou rectangulaire, puis par une déconstruction effective du format lui-même.

14 Notons néanmoins que cette dite déconstruction ne naît pas ex nihilo car, dès la Renaissance italienne et aux siècles suivants, certains peintres s'ingénient subtilement à désigner, à montrer la couture sémiotique. Nous pouvons voir cette ambiguïté entre le monde réel et celui de la représentation dans l'Annonciation ${ }^{7}$ de Francesco del Cossa ${ }^{8}$ par exemple, étudiée par Daniel Arasse, où un escargot anormalement gros se promène sur le cadre (19). L'escargot peint sur le bord inférieur du tableau semble se déplacer sur le cadre si bien que la notion même de cadre est subvertie :

L'escargot serait là pour figurer le caractère proprement figuratif de cette Annonciation. Il signale «le lieu d'entrée de ce tableau dans le tableau », lieu qu'il montre et démontre à la fois. La ligne de terre, le bord horizontal inférieur du tableau est celui qui semble le plus proche de l'observateur. Il implique directement et indirectement l'homme dans le dispositif de représentation et d'énonciation dont le cadre est l'élément moteur par le biais du cadre-cornice et de la couture sémiotique. (Charbonnier 174)

Dans la seconde moitié du XIXe siècle, nous voyons apparaitre cette même clôture sémiotique, dans certaines peintures, notamment dans le tableau d'Edouard Manet, Le balcon (1868-1869) ${ }^{9}$. "L'œuvre de Manet inaugure une époque où il s'agit de donner à voir la peinture » (Foucault 65). En effet, sa peinture fait apparaître la nature même du support, c'est-à-dire la matérialité fondamentale de sa constitution: sa structure rectangulaire. Michel Foucault nous montre, à propos du tableau Le balcon, le 
redoublement de la structure géométrique dans la peinture elle-même. L'aire géométrique de la toile s'auto-désigne en tant que surface/rectangle :

Un tableau qui est architecturé très manifestement par des lignes verticales et horizontales. La fenêtre elle-même redouble très exactement la toile et reproduit ses verticales et ses horizontales. Le balcon qui est devant la fenêtre, reproduit là encore les verticales et les horizontales [...]. Si vous ajoutez à cela ces persiennes que vous ne voyez pas, vous voyez que tout le tableau est encadré par ces verticales et ces horizontales. Loin que Manet ait voulu faire oublier le rectangle sur lequel il peignait, il ne fait que de le reproduire, d'insister sur lui, de le redoubler, de le multiplier à l'intérieur même de son tableau. (41)

Pour Michel Foucault, il s'agit de démontrer que dès l'œuvre de Manet, la modernité est présente : la surface rectangulaire, les axes horizontaux et verticaux sont à nouveau réinvestis comme tels. Nous assistons à une réinsertion de la matérialité de la toile, dans ce qui est représenté. D'autant que, dans cette peinture, Le balcon, la profondeur est esquivée alors même qu'il s'agit d'une fenêtre ouverte. En effet, cette dite fenêtre ouvre sur quelque chose d'entièrement obscur, de noir. Cela a pour conséquence de ramener le regard du spectateur vers les personnages, le balcon, et donc la surface de la toile, la réalité matérielle, bidimensionnelle. Manet signe ici la fin des pouvoirs illusionnistes de la représentation. En donnant consistance à la surface matérielle qu'est le tableau, il nie qu'il s'agisse d'une fenêtre ouverte sur le monde; il rompt avec l'illusion d'un creusement.

\section{Autoréflexivité de la surface carrée ou rectangulaire}

17 Au regard de notre modernité, et plus spécifiquement dans certaines œuvres du XX siècle, nous assistons à une réinsertion de la matérialité de la toile, en tant que surface carrée ou rectangulaire, structurée d'axes verticaux et horizontaux. Car une toile est bien une surface qui a une horizontale et une verticale. Ce format géométrique de la toile va être rendu visible, lisible, par une sorte d'autoréflexivité de la surface géométrique. Pour Jean Clay, la peinture du début $\mathrm{XX}^{\mathrm{e}}$ siècle s'identifie à son support bidimensionnel; elle assume sa planéité, sa frontalité. "C'est la logique interne du tableau qui dicte la forme - et non plus le monde extérieur. De proche en proche, l'organisation des surfaces l'emporte sur toute profondeur figurée»(172). C'est l'économie structurelle $\mathrm{du}$ tableau qui l'emporte sur la logique figurative/ représentative. « Il y a effacement des signes référentiels au profit des lois internes de l'organisme pictural » (173).

Prenons l'exemple de trois œuvres très connues au $\mathrm{XX}^{\mathrm{e}}$ siècle, qui valident clairement le discours de Jean Clay à propos de la modernité et qui mettent en évidence la matérialité rectangulaire de la toile : le fameux Carré blanc sur fond blanc, de Kasimir Malévitch, datant de 1918; les compositions de Piet Mondrian, à partir des années 1920 ; et l'incontournable série de Josef Albers, Homage to the square (1949-1976).

19 En effet, l'œuvre majeure de Kasimir Malévitch, Carré blanc sur fond blanc ${ }^{10}$, nous met en présence de cette problématique. L'artiste en a fait plusieurs versions, dont une est exposée au MOMA de New York (il aurait effectué en tout sept carrés blancs dont deux ont disparu). Avec cette peinture, nous sommes devant une "table rase ", une sorte de " degré zéro » de la peinture. Ce tableau est une complète abstraction, un rejet total de tout système figuratif, de toute norme académique. Malévitch aboutit à cette peinture par dépouillement, réduction, ascétisme. Il supprime toute forme jusqu'à ne garder 
qu'un seul carré, ultime, archétypal. Il supprime aussi tout système chromatique et le remplace par une présence, un aplat " a-chrome » : le blanc. Bien qu'il y ait une subtile nuance de blanc entre le carré et le fond, nous voyons apparaître un aspect monochromique. Dans son ascétisme formel et chromatique, cet artiste se veut radicalement avant-gardiste et projette sur la toile une sorte de "monde sans objet ».

Dans ce Carré blanc sur fond blanc, comme le titre l'indique, nous voyons affleurer à la surface, un carré incliné dans une position dynamique (une des caractéristiques du Futurisme qui avait influencé Kasimir Malévitch, un temps), une sorte de carré dans le carré. En effet, le carré géométrique est rejoué, répété, dans l'espace/surface blanc de la toile. Comme si le fond entourait le carré blanc, tel un cadre peint autour de la forme carrée. De fait, ce carré dans le carré, ce cadre dans le cadre, engendre une autoréflexivité de la surface carrée qui, de fait, s'auto-désigne en tant que surface plane. De plus, au-delà de l'abstraction, ce peintre ouvre un nouveau territoire encore non pratiqué à l'époque, celui de la monochromie. Il étend aussi le champ des possibles concernant la forme carrée qui, à partir des années 1920, va devenir omniprésente, figure archétypale et sous-jacente à toute la modernité du XXe siècle.

En effet, la figure du carré rend lisible la matérialité géométrique du support pictural et, au-delà, joue un rôle très important concernant la notion même de formalisme. Cette conception antinaturaliste, fondée sur l'autonomie des propriétés formelles spécifiques à chaque médium, affirme leur caractère auto-suffisant dans la genèse de notre expérience, au regard de l'œuvre. La forme carrée serait, dès lors, une instance performante mettant en évidence un principe d'autonomie radicale des formes artistiques. La théorie formaliste, par l'intermédiaire de cette figure, essaie d'identifier un art à son médium : pour la peinture, le caractère bidimensionnel du plan.

De ce point de vue, l'œuvre de Piet Mondrian fait écho, elle aussi, à cette problématique. En effet, ce peintre met en place une série de compositions à partir des années 1920. Ces peintures strictement abstraites sont réduites à des invariants spécifiques, notamment des aplats carrés ou rectangulaires de couleurs primaires (jaune, bleu, rouge) ainsi que le blanc qui peut aller jusqu'au gris clair. Ces aplats sont cadrés par des bandes horizontales et verticales noires plus ou moins larges. Le tout est orthogonal, puisque Piet Mondrian voulait dépouiller ces peintures de toute « anecdote » (selon ses propres termes), à savoir, la courbe et l'oblique. À partir de ces invariants, une variabilité infinie se met en place.

Si l'aventure artistique s'opère donc selon un processus fortement répétitif, il faut l'entendre comme une répétition ouverte qui comprend de la différence, susceptible d'engendrer non pas du même mais de l'autre. Il s'agit d'un processus paradoxal : c'est parce que l'on refait que l'on fait autre chose. (Barral 31)

La sérialité débouche sur la notion d'œuvre ouverte (Eco), de création qui en appelle toujours une autre. Nous voyons poindre ici la tension entre la notion de finitude (le chef d'œuvre) et celle d'infini (la série). En fait, la sérialité va commencer à se systématiser, à devenir un enjeu fort, à partir de l'œuvre de Claude Monet, c'est-à-dire au début de la modernité. Le chef-d'œuvre académique, se voulant unique, parfait et indépassable, va être battu en brèche, anéanti par l'enjeu de la sérialité puisque la série se construit, se structure, s'élabore en tant que variation infinie, œuvre multiple et non pas unique. Elle est gouvernée par les règles de la variation, réglée par le principe d'équivalence, et décline les jeux subtils d'une répétition qui engendre de la différence. Gilles Deleuze écrit à ce propos : « La différence est comprise dans la répétition [...]. Les 
variantes expriment plutôt des mécanismes différentiels qui sont de l'essence et de la genèse de ce qui se répète » (28).

De plus, au-delà de l'enjeu de la sérialité, l'orthogonalité radicale des œuvres abstraites de Piet Mondrian met en évidence la couture sémiotique rectangulaire : le rectangle géométrique de la surface/toile est rejoué, divisé, multiplié à l'intérieur de la structuration du tableau, d'autant que les bandes noires séparant les couleurs accentuent un peu plus l'effet de répétition du cadre dans le cadre. Ces bandes forment comme une sorte de "grille » qui barre l'espace diégétique de la toile. Le peintre ne va pas au-delà de la surface bidimensionnelle. Il n'essaie plus de donner l'illusion d'un creusement mais, bien au contraire, assume la planéité du format et sa surface rectangulaire. À propos de la grille, Rosalind Krauss déclare que

[s]patialement, la grille affirme l'autonomie de l'art, bidimensionnelle, géométrique, ordonnée, elle est anti-naturelle, antimimétique, et s'oppose au réel. C'est à quoi l'art ressemble lorsqu'il tourne le dos à la nature. Par la planéité qui résulte de ses coordonnées, la grille permet de refouler les dimensions du réel et de les remplacer par le déploiement latéral d'une seule surface. L'entière régularité de son organisation est le résultat, non de l'imitation, mais d'un décret esthétique. (94)

Un peu plus tard, à partir de 1949, une autre œuvre rejoue l'autoréflexivité de la surface carrée : il s'agit de la série Homage to the square de Josef Albers. C'est une série qui arrive tardivement dans la vie du peintre (puisqu'il a déjà 61 ans) mais qui va le faire connaître internationalement. Cette série, réalisée jusqu'à sa mort en 1976, fait partie d'une étude systématique des couleurs. Josef Albers est d'ailleurs l'auteur d'un livre célèbre chez les plasticiens, L'Interaction des couleurs. Par l'intermédiaire de cet hommage au carré, il essaie d'étudier d'une manière quasi systématique les effets optiques des rapports de différentes couleurs, en choisissant une même structure afin de l'aider à focaliser son attention sur son sujet principal, la couleur. Cette série d'œuvres représente trois ou quatre carrés imbriqués, selon une disposition rigoureusement identique : seules les couleurs changent. Et quel que soit le support (toile, bois, ou papier), chaque œuvre obéit à une formule invariable : un format carré (de 40,6 x 40,6 cm jusqu'à 122 x $122 \mathrm{~cm}$ ). Cette structuration est reprise d'une manière identique dans toute la série afin de mieux mettre en évidence les rapports, les jeux optiques des couleurs. Les harmonies de couleur deviennent l'enjeu principal. Mais, en choisissant la forme carrée et en la multipliant plusieurs fois, comme une sorte de cadre dans le cadre, ou de tableau dans le tableau, Josef Albers met aussi en scène la couture sémiotique de la toile : la géométrie carrée devient omniprésente car répétée dans une sorte de mise en abyme. Homage to the square est effectivement, comme son nom l'indique, un hommage à l'omniprésence du carré, non seulement dans l'œuvre de cet artiste mais aussi dans toute la modernité du XXe siècle. Les peintres assument enfin pleinement la toile en tant que surface : le cadre géométrique matriciel et opératoire devient un jeu de formats carrés.

\section{Déconstruction du format carré ou rectangulaire}

26 À partir des années 1960, les peintres vont aller encore plus loin puisqu'une déconstruction devient effective. La toile elle-même fait l'objet de cette ultime déconstruction. C'est notamment le cas dans le mouvement Supports/Surfaces : une même volonté unit les artistes de ce mouvement, celle d'interroger l'objet-tableau. 
Adoptant une logique déconstructive, ils en analysent les éléments constitutifs : le support (châssis), la surface (toile) et la matière (peinture).

Prenons l'exemple de Claude Viallat, l'un des fondateurs du mouvement Supports/ Surfaces. Au cours de l'été 1966, il met au point un procédé systématique que nous pourrions aussi appeler « outil visuel » : une même forme ressemblant à un osselet ou une éponge, apposée en imprégnation sur la toile non tendue et non apprêtée. Viallat remet en cause le support en séparant la toile du châssis. La toile est dès lors libérée de sa forme rectangulaire et le peintre introduit des supports inhabituels qui peuvent être neufs ou recyclés, tels que des rideaux, bâches, parasols, tentes, chemises. Ces différents matériaux ont une incidence sur l'œuvre par leur matière, leur densité, leur coloration. Cet artiste met en place, dès lors, un système sériel à partir d'un invariant spécifique (son « outil visuel »). Sans annihiler complètement le format rectangulaire, les toiles peuvent prendre différentes formes: non tendues, plissées, ondulantes, triangulaires, allongées, ovales, déformées. Comme l'écrit Jean-Charles Lebahar :

La peinture américaine des années soixante a influencé Viallat comme tous ceux de sa génération, en exhibant des travaux dont la plupart n'avait plus rien à voir avec la peinture de chevalet (désolidariser la toile du châssis, positionner la toile dans l'espace autrement que dans la verticalité - par exemple au sol. Utiliser de nouvelles matières, de nouveaux supports, assembler des objets banals à des toiles peintes). (15)

Concernant maintenant l'œuvre de Daniel Dezeuze, la matérialité du support est mise en évidence par l'intermédiaire du châssis qui est exposé seul. Cet artiste, faisant lui aussi partie de Supports/Surfaces, prend ici le contrepied des œuvres de Viallat. En effet, en 1967, Dezeuze expose son désormais célèbre Châssis ${ }^{11}$ : il s'agit d'une feuille plastique transparente (qui remplace la toile) tendue sur un châssis en bois. Dès lors, la toile disparaît et le châssis apparaît. Donc, ce qui est offert au regard du spectateur, c'est en quelque sorte "l'envers du décor » : la matérialité du support, la structuration de l'œuvre est rendue entièrement lisible, mettant à nu les éléments matériels qui composent le tableau. Le support visible du châssis et la surface transparente du plastique remplacent l'espace illusionniste de la toile, et militent pour une perception didactique de la démonstration, qui se transforme en déconstruction. Cette œuvre de Daniel Dezeuze se trouve posée au sol, contre un mur, en position contraire à un accrochage classique : le châssis ne fonctionne plus comme une toile mais comme un objet, tel un ready-made posé contre le mur. Le tableau, objet omniprésent dans l'art occidental depuis des siècles, se retrouve exposé dans son entière matérialité. De fait, l'artiste en propose une lecture à la fois matérielle et ironique.

\section{Jeux de formats}

Toujours dans les années 1960, certains artistes, notamment aux Etats-Unis, vont choisir d'autres manières de remettre en cause la norme rectangulaire de la toile. C'est le cas notamment de Frank Stella. Cet artiste met en œuvre des découpes de toiles non rectangulaires. Il est le premier chez qui une relation de nécessité s'établit entre les contours extérieurs du format et sa structure intérieure. Stella crée des formes différentes par une simple soustraction tout en donnant le sentiment qu'il a agrandi un détail : la géométrie de la toile est à la fois rejouée et revisitée et, de fait, devient très visible. 
Reprenant la logique interne des toiles noires, Stella s'appliqua ensuite à modifier la forme extérieure de la toile, avec des formats inhabituels comme celui d'Orphir (1960-1961). Par ailleurs, autour du milieu des années 1960, il employa un châssis épais pour décoller le tableau du mur, à la façon d'un relief. (Moszynska 202) des portions considérables qui seront ôtées aux carrés ou rectangles virtuels par ces œuvres. Il ne restera selon les cas qu'un L inversé, un large U, ou une croix grecque » (Pacquement 40). La toile, évidée à certains endroits, fait dès lors l'objet d'une découpe non rectangulaire et la production de l'artiste se transforme en un jeu de formats inattendus, surprenants, non conventionnels. La couture sémiotique de la toile devient tout d'un coup extrêmement visible, car inhabituelle. La modernité de la seconde moitié du XXe siècle n'hésite plus à mettre en évidence la matérialité géométrique du tableau tout en jouant efficacement avec la découpe iconoclaste de la surface.

31 Un autre artiste américain, Ellsworth Kelly, va opter pour des formes ainsi que des assemblages non rectangulaires, et ce à peu près à la même époque que Franck Stella. E. Kelly a fréquemment recours à des châssis qui transgressent l'orthogonalité imposée par les usages esthétiques et sociaux de l'objet-tableau. Il va notamment expérimenter la forme courbe, mettant en place des séries de peintures monochromes non rectangulaires. De plus, à partir des années 1970, il aura souvent recours à des assemblages de monochromes (que nous pourrions dire aussi «juxtaposés ») qui euxmêmes forment un tout non rectangulaire. En jouant avec différentes sortes d'assemblages, Kelly rend enfin visible la géométrie du tableau. Et c'est bien le propre de la modernité que de rendre visible et autonome la forme et la couleur. Nous ne sommes plus dans un processus d'autonomisation (comme par exemple, le Fauvisme du début $\mathrm{XX}^{\mathrm{e}}$ siècle) mais au contraire dans une autonomie totale que l'histoire de la peinture abstraite n'a fait qu'accentuer.

Une riche dialectique entre bords et surface, couleur, forme, et masse, formes peintes sur fond peint, panneaux monochromes, seuls, accolés ou distribués sur le mur [...], découpes asymétriques, elliptiques, se déclinant à deux ou en nombre, jouant des lignes tendues, droites ou courbes, ou bien une combinaison des deux. (Hindry 57-58)

Les œuvres créées par Ellsworth Kelly sont des objets totalement autonomes qui dépassent le cadre spécifiquement rectangulaire de la peinture, et peuvent s'orienter du côté du volume, et donc, de l'objet sculptural. Quel que soit l'objet en question, les surfaces ne présentent ni incident, ni trace de pinceau, afin que le spectateur focalise avant tout son attention sur la forme et la couleur. Nous assistons dès lors à une libération totale de la forme des limites imposées par le tableau afin qu'elle puisse exister de façon autonome, comme dans le Blue Red Rocker (1963) en aluminium : la peinture se transforme en un objet / volume.

\section{Conclusion}

Nous avons émis l'hypothèse d'un format normatif et rectangulaire de la toile datant spécifiquement de la Renaissance italienne, et de sa déconstruction au regard de notre modernité. Cette évolution s'est fortement accélérée dans la seconde moitié du XX siècle, remettant même en cause la peinture en tant que médium, allant au-delà d'une simple déconstruction. Car dans les années 1970, le récit historiographique est associé avant tout au Land Art, au body art, à l'art vidéo, aux performances et à la 
photographie, c'est-à-dire à des pratiques qui rompent avec l'objet-tableau. Certains théoriciens déclarent la "fin de la peinture » (discours très opérant dans les années 1970 et 1980$)^{12}$, se mettant en porte-à-faux face au médium/peinture qui se renouvelle complètement à partir des années 1980, et ce, grâce à différents mouvements picturaux européens et internationaux. La peinture fut néanmoins l'objet d'un dénigrement régulier, étant considérée au mieux comme anachronique, au pire comme totalement dépassée.

Contrairement au formalisme lié à la Modernité, la peinture contemporaine est souvent intégrée dans des propositions hybrides. La grande majorité des artistes contemporains ne semblent plus à la recherche d'une forme incréée, libre de toute ressemblance ou de tout modèle et qui se réduirait à quoique ce soit d'autre qu'elle-même. Bien au contraire, ceux-ci s'identifient à des propositions impures, métissées, et la plupart du temps truffées de références et clins d'œil à l'histoire de l'art.

\section{BIBLIOGRAPHIE}

\section{Ouvrages cités}

ALBERS, Josef. Interaction of Color. New Haven : Yale University Press, 1963.

ALBERTI, Leon Battista. De la Peinture (De Pictura). 1435. Paris : Macula/dédale, 1992.

ARASSE, Daniel, On n'y voit rien. Paris : Denoël, 2000.

BARRAL, Jacqueline et al. Figures de la répétition. Travaux LXXV. Saint-Etienne : Presses

Universitaires de Saint-Etienne, 1992.

CAHN, Isabelle. Le cadre des peintres. Paris : Hermann, 1989.

CHARBONNIER, Louise. Cadre et regard. Généalogie d'un dispositif. Paris : L'Harmattan, 2007.

CLAY, Jean. De l'impressionnisme à l'art moderne. Paris : Hachette, 1975.

DELEUZE, Gilles. Différence et répétition, Paris, Presses Universitaires de France, 1968.

ECO, Umberto. L'œuvre ouverte, Paris, Seuil, 1965.

FOUCAULT, Michel. La peinture de Manet. Paris : Seuil, 2004.

GOETZ, Adrien. « Encadrement des œuvres, histoire de l'art occidental ». Encyclopcedia Universalis. URL : http://www.universalis-edu.com/encyclopedie/encadrement-des-oeuvres-histoire-de-lart-occidental/_consulté le 15 avril 2021).

HINDRY, Ann. « Kelly, vivre l'espace de la couleur ». Ellsworth Kelly. Paris : Fondation Louis Vuitton \& Manuella Éditions, 2014. 57-58.

JIMENEZ, MARC. La Querelle de l'art contemporain, Paris, Gallimard, 2005.

JOLY, Martine. L'image et les signes. Paris. Armand Colin, 2008. 
KRAUSS, Rosalind. L'originalité de l'avant-garde et autres mythes modernistes, Paris, Macula, 1993.

LEBAHAR, Jean-Charles. Claude Viallat. Une issue à travers le mur, Paris, Au même titre, 1999.

MOSZYNSKA, Anna. L'Art abstrait, Paris, Thames \& Hudson, 1998.

PACQUEMENT, Alfred. Frank Stella. Paris : Flammarion, 1988.

SHAPIRO, Meyer. Style, artiste et société. Paris : Gallimard, 1982.

SOURIAU, Etienne. Vocabulaire d'esthétique, Paris, Presses Universitaires de France, 1990.

TRESPEUCH, Hélène. La crise de l'art abstrait ? Récits et critique en France et aux Etats-Unis dans les années 1980, Rennes, Presses Universitaires de Rennes, 2013 ;

\section{Fuvres citées}

ALBERS, Josef. Homage to the square. 1949-1976. Série de peintures sur bois ou sur toile allant de 40,6 x 40,6 cm à 122 x $122 \mathrm{~cm}$.

DE MESSINE, Antonello. Saint-Jérôme dans un intérieur. 1474-1475. Peinture à l'huile sur panneau de bois. 45,7 x 36,2 cm. $\mathrm{N}^{\circ}$ d'inventaire : NG1418. National Gallery, Londres.

DEL COSSA, Francesco. Annonciation. Entre 1470 et 1472. Tempera sur panneau de bois. 139 x 113,5 $\mathrm{cm} . \mathrm{N}^{\circ}$ d'inventaire : AM-43-44-PS01. Gemäldegalerie Alte Meister, Dresde, Allemagne.

DEZEUZE, Daniel, Châssis, 1967. Feuille de plastique transparent tendue sur châssis. 194,5 x 130 x 2 cm. $\mathrm{N}^{\circ}$ d'inventaire : AM 1985-173. Centre Georges Pompidou, Paris, France.

MALÉVITCH, Kasimir. Carré blanc sur fond blanc. 1918. Peinture à l'huile sur toile. 79,4 x 79,4 cm. $\mathrm{N}^{\circ}$ d'inventaire : 817.1935. Museum of Modern Art, New York.

MANET, Edouard : Le balcon. 1868-1869. Peinture à l'huile sur toile. 170 x $124 \mathrm{~cm} . \mathrm{N}^{\circ}$ d'inventaire : RF 2772. Musée d'Orsay, Paris.

\section{NOTES}

1. La forme rectangulaire suppose en effet des informations implicitement admises : notamment la posture frontale du spectateur face à l'image, impliquant une certaine distance afin que la lecture de cette dernière puisse s'effectuer. Dès lors, il est implicite que la géométrie carrée ou rectangulaire indique le cadre d'une diégèse.

2. Le cadre-objet est une bordure (formant un carré à l'origine) entourant un tableau ou un miroir. Cet objet influence ladite monstration de l'image, souligne l'aire géométrique de cette dernière, et se situe dans une histoire spécifique de l'encadrement, en Occident. Ce cadre-objet sera remis en cause dès la fin du XIX ${ }^{\mathrm{e}}$ siècle au moment où la Modernité s'affirme contre l'académisme.

3. Nous distinguerons au fur et à mesure de notre discours l'objet/toile, de l'objet/ cadre.

4. La notion d'image, ici, renvoie à l'image en tant qu'objet: dessin, peinture, photographie ou film... Il s'agit d'un artefact représentant, sur un support, quelque chose de réel ou de possiblement réel. Cette image/objet est à distinguer de l'image strictement mentale. 
5. Alberti Leon Battista (1404-1472) est un des grands humanistes de la Renaissance italienne $\mathrm{du} \mathrm{XV}$ siècle ; théoricien, peintre, sculpteur, architecte, et mathématicien. Il fut un important théoricien de la peinture du Quattrocento, avec son fameux livre De pictura, datant de 1435.

6. De Messine Antonello, Saint-Jérôme dans un intérieur, 1474-1475. Peinture à l'huile sur panneau de bois. 45,7 x 36,2 cm. Nº d'inventaire : NG1418. National Gallery, Londres, Royaume-Uni.

7. Francesco Del Cossa Annonciation, entre 1470 et 1472. Tempera sur panneau de bois. 139 x 113,5 cm. No d'inventaire : AM-43-44-PS01 and Gal.-Nr. 43. Gemäldegalerie Alte Meister, Dresde, Allemagne.

8. Francesco Del Cossa (1436-1478) : peintre italien important, concernant la période du Quattrocento. Il fut notamment influencé par les œuvres de Donatello, Piero della Francesca, et Andrea Mantegna.

9. Edouard Manet : Le balcon, 1868-1869. Peinture à l'huile sur toile. 170 x $124 \mathrm{~cm} . \mathrm{N}^{\circ}$ d'inventaire : RF 2772. Musée d'Orsay, Paris, France.

10. Kasimir Malévitch, Carré blanc sur fond blanc, 1918. Peinture à l'huile sur toile. 79,4 $\mathrm{x}$ $79,4 \mathrm{~cm} . \mathrm{N}^{\circ}$ d'inventaire 817.1935. Museum of Modern Art, New York, USA.

11. Daniel Dezeuze, Châssis, 1967. Feuille de plastique transparent tendue sur châssis. 194,5 x 130 × 2 cm. N d'inventaire: AM 1985-173. Centre Georges Pompidou, Paris, France.

12. Dans son ouvrage, La crise de l'art abstrait ? Récits et critique en France et aux Etats-Unis dans les années 1980, Rennes, Presses universitaires de Rennes, 2013), Hélène Trespeuch parle de certains théoriciens qui ont eu tendance à négliger voire à rejeter le médium / peinture, notamment, Douglas Crimp, Thomas Lawson, ou Lucy Lippard. Dans ce même ouvrage, l'auteure cite le livre de Marc Jimenez, La Querelle de l'art contemporain (Paris, Gallimard, 2005), qui évoque lui aussi, cette même problématique, à savoir, le rejet effectif de la peinture chez certains théoriciens et plasticiens des années 1970 et 1980.

\section{RÉSUMÉS}

Rarement questionné, le dispositif iconique du cadre rectangulaire informe la plupart des images qui font notre actualité (photographies, cinéma, vidéos, écrans...). La présupposition de la forme quadrangulaire semble avoir son origine dans l'apparition, à la Renaissance italienne, du tableau moderne. Le cadre rectangulaire conditionne en effet notre réception de l'œuvre. De par la modernité en général, et plus particulièrement dans les œuvres abstraites du XX⿳⺈冂大 siècle, nous assistons à une réinsertion de la matérialité de la toile en tant que surface carrée ou rectangulaire. Ce format géométrique de la toile va être rendu visible par une sorte d'autoréflexivité de la surface géométrique comme dans l'œuvre de Piet Mondrian ou plus tard celle de Josef Albers. La toile du peintre va ensuite faire l'objet d'une déconstruction et d'un jeu de formats, notamment avec le mouvement artistique français Supports / Surfaces, à la fin des années 60 , ainsi que chez certains artistes américains. 
Rarely questioned, the iconic device of the rectangular frame informs most of the images that make our news (photographs, cinema, videos, screens...). The presupposition of the quadrangular form seems to have its origin in the introduction of the modern framed painting in the Italian Renaissance. The rectangular frame conditions indeed our reception of the artwork. As a result of modernity in general, and more particularly in the abstract works of the $20^{\text {th }}$ century, we are witnessing a reintegration of the materiality of the canvas as a square or rectangular surface. This geometric format of the canvas is made visible by a kind of self-reflexivity of the geometric surface as in the work of Piet Mondrian or later that of Josef Albers. The painter's canvas is then the object of a deconstruction and a play with formats, notably with the French artistic movement Supports / Surfaces, at the end of the 60s, as well as with some American artists.

\section{INDEX}

Keywords : canvas, frame, normative format, iconic device, rectangular geometry, Renaissance, modernity, self-reflexivity, deconstruction, play with formats

Mots-clés : toile, cadre, format normatif, dispositif iconique, géométrie rectangulaire, Renaissance, modernité, autoréflexivité, déconstruction, jeux de formats

\section{AUTEUR}

\section{FRÉDÉRIC MONTÉGU}

Université Lyon 2, Laboratoire Passages Arts \& Littératures / XX-XXI.

Frédéric Montégu est professeur agrégé d'arts plastiques, docteur en esthétique et sciences de l'art et qualifié aux fonctions de Maître de conférences (sections 22 \& 18). Il enseigne l'esthétique et l'histoire de l'art à l'université Lumière Lyon 2 (département histoire de l'art \& archéologie). Membre associé au laboratoire Passages Arts \& Littératures / XX-XXI, de l'Université Lumière Lyon 2, il est l'auteur d'un ouvrage en deux volumes sur l'œuvre de Mark Rothko : Image et Abstraction dans l'œuvre de Mark Rothko (Paris, L'Harmattan, 2014). Frédéric Montégu continue à interroger l'œuvre de cet artiste, ainsi que l'expressionnisme abstrait américain, tout en ouvrant son champ d'investigation sur des œuvres contemporaines (Bernard Piffaretti, Wolfgang Laib, Chiharu Shiota, notamment). 